\title{
Mass Spectra of Some Organo-Lead and Organo-Mercury Compounds
}

\author{
Vernon H. Dibeler and Fred L. Mohler
}

\begin{abstract}
Mass spectra of tetramethyl and tetraethyl lead and of dimethyl, diethyl, and di- $n$-butyl mercury were obtained with a Consolidated 21-102 mass spectrometer. Monoisotopic spectra were calculated by using the isotopic abundance derived from each spectrum. A variation in the lead isotope abundance was observed in the commercially available lead alkyls. No variation was observed among the mercury alkyls and good agreement was obtained with previously published measurements on mercury vapor. There was no indication of voltage discrimination. The mass spectra of the lead alkyls are qualitatively similar. The principal portion of the total ionization is contributed by the dissociation of one and three alkyl groups. Ions resulting from the dissociation of one or more hydrogen atoms are small, as are the hydrocarbon ions. Hydrides and hydrocarbon ions resulting from intramolecular rearrangement were observed. The principal part of total ionization in the mercury alkyl spectra was contributed by hydrocarbon ions. Hydrides and other rearrangement peaks were observed. A preliminary experiment on a leaded gasoline, using a model 21-103 spectrometer, indicated that sensitivity is sufficient to analyze for a few hundredths of a mole percent of tetraethyl lead with a precision of about 2 percent of the value.
\end{abstract}

\section{Introduction}

In recent years hydrocarbons have been an important subject of fundamental and applied research $[1]^{1}$ in mass spectrometry, as well as the principal subject of the systematic accumulation of mass spectral data [2]. It is surprising, however, that very little has been reported on the mass spectra of organometallic compounds in spite of the interesting properties and commercial importance of some of these materials.

The volatile alkyl compounds of many of the elements, including zinc, germanium, tin, and lead were used by Aston [3] in his early studies of the isotopic constitution of the elements. No details of the mass speetra were reported, but it was subsequently shown by various investigators that corrections as to the identity and abundance of the isotopes so obtained were necessary because of the presence of hydrides in the mass spectra.

More recently, Hipple and Stevenson $\lceil 4\rceil$ studied the ionization by electron impact of methyl and ethyl radicals formed in the mass spectrometer by the thermal decomposition of tetramethyl and tetraethyl lead. They did not report the complete mass spectra of the undecomposed molecules but observed that in both cases the molecule ion abundance was almost immeasurably small. A rough measurement using $15-\mathrm{v}$ electrons and an ion source at about room temperature showed the trimethyl lead ion to be about 50 times as abundant as the tetramethyl lead ion. No similar measurement was given for tetraethyl lead.

There have been a number of mass spectrometric studies of the isotopic abundance of lead obtained from various natural and commercial sources. Nier [5] has demonstrated a wide variation in the isotopic abundance of leads obtained from virgin

\footnotetext{
1 Figures in brackets indicate the literature references at the end of this paper.
}

ores. Lead obtained from commercial sources, sometimes called "common lead", may be expected to show much less variation than the natural ores because it is a mixture from many sources. However, one would not expect isotopic ratios to be accurately constant in compounds made at different times or in different places. Recent investigators have measured lead isotopes by using inorganic lead halides, which preclude the formation of hydrides but present technical difficulties not encountered in the use of the relatively volatile alkyl compounds.

Isotopic analyses of mercury have usually been made by using the vapor of the element $[6,7]$. Experimental difficulties again result from the low vapor pressure of the element and from the possibility of amalgamation with interior parts of the mass spectrometer. No measurable variation in the natural abundance of the mercury isotopes has been reported.

It is the purpose of this paper to give a complete description of the mass spectra of several related organometallic compounds and to demonstrate the applicability of these molecules to isotopic analysis. A discussion is also given concerning the dissociation processes of these molecules.

\section{Experimental Details}

Mass spectra were obtained by using a Consolidated 21-102 mass spectrometer with the resolving power increased to about 1 in 350 . Liquid samples were introduced by means of a micropipet through a porous glass disk covered with liquid gallium. The pressure of the expanded vapors was measured with a micromanometer [8]. Spectra were obtained by varying the ion accelerating voltage. Each complete spectrum required two magnetic field settings: $0.535 \mathrm{amp}$ for the mass range 12 to 90 , and 1.320 amp for the mass range 48 to 350 . The correlation 
of the two mass ranges was made by the comparison of peak heights in the overlapping mass range.

The preliminary test of the analysis of a leaded gasoline of known composition ${ }^{2}$ was made after the above instrument was modified to a model 21-103. The total pressure of the sample introduced was measured with the micromamometer. The partial pressure of the lead tetraethyl was calculated from the observed ion current of the $\mathrm{Pb}\left(\mathrm{C}_{2} \mathrm{H}_{5}\right)^{+}$ion and the previously measured sensitivity (ion current per unit of pressure) of this ion.

The tetramethyl and tetraethyl lead were obtained from the Ethyl Corp. Portions of the liquid samples were removed from the shipping containers and vacuum distilled without rectification into Pyrex ampoules, sealed in vacuum and protected from light until used. The tetramethyl lead (sample number 133.7-XP-3) was received sometime in 1947 and was considered to be of high purity. An estimated 0.1 mole percent of tetramethyl tin was the only impurity observed. The tetraethyl lead was received about the same time. Total impurities were estimated less than 2 mole percent and ascribed principally to ethyl chloride.

Dimethyl, diethyl, and di-n-butyl mercury were obtained from the Eastman Kodak Co. These were stored in vacuum in the same manner as the lead compounds. They were not as pure as the lead compounds and contained unknown small amounts of oxygenated molecules, alkyl halides, and possibly hydrocarbons. Impurities with vapor pressures considerably different from that of the alkyl mercury compounds were removed in the process of distilling the samples into the storage ampoules.

\section{Results}

Table 1 gives the principal part of the polyisotopic spectrum of tetramethyl lead for $70-\mathrm{v}$ electrons. The contributions of ions containing $\mathrm{C}^{13}$ atoms have been subtracted. The abundances of the ions are

TABLE 1. Polyisotopic spectrum of tetramethyl lead for $70-v$ electrons

\begin{tabular}{|c|c|c|c|}
\hline$m / e$ & $\begin{array}{l}\text { Relative in- } \\
\text { tensities }\end{array}$ & $m / e$ & $\begin{array}{l}\text { Relative in- } \\
\text { tensities }\end{array}$ \\
\hline $\begin{array}{r}12 \\
13 \\
14 \\
15 \\
16 \\
\\
204 \\
205 \\
206 \\
207 \\
208 \\
209 \\
\\
216 \\
217 \\
218 \\
219 \\
220 \\
221 \\
222 \\
223 \\
224\end{array}$ & $\begin{array}{c}0.32 \\
.75 \\
2.59 \\
12.2 \\
0.25 \\
\\
1.65 \\
0.15 \\
33.2 \\
28.5 \\
66.2 \\
5.73 \\
0.03 \\
.08 \\
.62 \\
3.98 \\
5.13 \\
49.6 \\
40.3 \\
85.3 \\
0.40\end{array}$ & $\begin{array}{l}230 \\
231 \\
232 \\
233 \\
234 \\
235 \\
236 \\
237 \\
238 \\
239 \\
\\
248 \\
249 \\
250 \\
251 \\
252 \\
253 \\
254 \\
\\
266 \\
267 \\
268\end{array}$ & $\begin{array}{c}0.02 \\
.09 \\
.09 \\
.17 \\
.66 \\
.35 \\
13.1 \\
10.6 \\
24.9 \\
0.25 \\
.03 \\
2.56 \\
0.50 \\
52.4 \\
41.2 \\
100.0 \\
0.31 \\
.10 \\
.08 \\
.21\end{array}$ \\
\hline
\end{tabular}

${ }^{2}$ Sample obtained from the Bureau's Engine Fuel Section. relative to the most abundant ion of mass 253 . Doubly charged ions appearing at masses $118,118.5$, $119,125.5,126$, and 126.5 are omitted from the table. Their abundance was 0.1 percent or less of the maximum peak. One metastable transition peak was observed with an apparent mass of 196.6 and a relative abundance of 0.22 percent. It was attributed to the delayed dissociation: $\left(253^{+}\right) \rightarrow$ $\left(223^{+}\right)+30$. The sensitivity of the 253 peak-attributed to the $\mathrm{Pb}^{208} \mathrm{C}_{3} \mathrm{H}_{9}{ }^{+}$ion-was about 0.7 times the sensitivity of the 43 peak of $n$-butane under similar conditions.

In order to compare the relative probabilities of the various dissociation processes in tetramethyl lead, it is first necessary to calculate the monoisotopic spectrum. This is possible if the relative abundances of the lead isotopes are known. For reasons that will be apparent in the following pages the isotopic abundances must be computed from the same mass spectrum. From the spectrum in the mass range 204 to 209 (table 1 ) it is obvious that hydride ions are present in addition to the atom ions. An approximation of the hydride abundance is given by the ratio $209 / 208$; namely, $5.73 / 66.2=0.087$. No dihydride was observed, and it is estimated that the ratio $\mathrm{PbH}_{2}{ }^{+} / \mathrm{Pb}^{+}$is less than 0.002 . Therefore, the approximate monohydride contribution to mass 208 is calculated from the 207 peak as $28.5 \times 0.087=2.2$. Thus the more accurate $\mathrm{PbH}+\mathrm{Pb}^{+}$ratio is given by $5.73 / 64.0=0.090$. Using this ratio, the hydride contributions were calculated and subtracted from the 207 and 208 peaks. The mole percentages of the $\mathrm{Pb}^{204}, \mathrm{~Pb}^{206}, \mathrm{~Pb}^{207}$, and $\mathrm{Pb}^{208}$ ions were computed. The first row of table 2 gives the mean values thus obtained for tetramethyl lead from four independent spectra. The mean deviation expressed in this and following tables is merely an indication of the reproducibility of the data. The estimated accuracy is 1 percent of the value for the principal isotopes and 5 percent for the rare isotope. Also included in table 2 are the isotopic abundance values for "common" lead reported by White and Cameron [9] and the extreme abundance values for lead ores reported by Nier [5].

The mean values for the isotopic abundance observed in tetramethyl lead were used in computing the monoisotopic spectrum, starting at the heavymass end of each ion group, by computing the contribution of each isotopic ion to the total ion peaks as illustrated by table 3 . Columns 1 and 2 repeat

TABLE 2. Mole percentage of lead isotopes

\begin{tabular}{|c|c|c|c|c|}
\hline Mass ... & 204 & 206 & 207 & 208 \\
\hline $\begin{array}{l}\mathrm{Pb}\left(\mathrm{CH}_{3}\right)_{4} \\
\text { Mean deviation. }\end{array}$ & $\begin{array}{r}1.31 \\
\pm .02\end{array}$ & $\begin{array}{l}26.79 \\
\pm .04\end{array}$ & $\begin{array}{l}20.55 \\
\pm .04\end{array}$ & $\begin{array}{l}51.35 \\
\pm .06\end{array}$ \\
\hline $\begin{array}{l}\mathrm{Pb}\left(\mathrm{C}_{2} \mathrm{H}_{5}\right)_{4} \\
\text { Mean deviation }\end{array}$ & $\begin{array}{r}1.36 \\
\pm .01\end{array}$ & $\begin{array}{l}25.42 \\
\pm .05\end{array}$ & $\begin{array}{l}21.11 \\
\pm .02\end{array}$ & $\begin{array}{l}52.10 \\
\pm .03\end{array}$ \\
\hline $\begin{array}{l}\text { White and Cameron [9]; } \mathrm{PbCl}_{2} \text {, } \\
\text { "common lead" }\end{array}$ & 1.37 & 25.15 & 21. 11 & 52. 38 \\
\hline $\begin{array}{l}\text { Nier [5]; } \mathrm{PbI}_{2} \text {, Galena, Ivigtut, } \\
\text { Greenland }\end{array}$ & 1.55 & 22.51 & 22.60 & 53.34 \\
\hline Galena, Joplin, Mo & 1. 23 & 27.52 & 19.81 & 51.43 \\
\hline
\end{tabular}


the $\mathrm{Pb}\left(\mathrm{CH}_{3}\right)^{+}$portion of the polyisotopic spectrum given in table 1 . The ion at mass 224 is entirely the result of $\mathrm{HPb}^{208} \mathrm{CH}_{3}+$ ions. The abundance of this ion (0.40) times the relative abundances of the lead isotopes (obtained from table 2) gives the contributions of the isotopic ions ${ }^{3}$ listed in column 3 , table 3 . The calculated contribution of the $\mathrm{HPb}^{207} \mathrm{CH}_{3}{ }^{+}$ion (0.16) subtracted from the observed 223 peak (85.3) gives the abundance of the $\mathrm{Pb}^{208} \mathrm{CH}_{3}{ }^{+}$ion (85.14). Again the relative abundance values of the lead isotopes are used to calculate the contributions of the isotopic $\mathrm{PbCH}_{3}{ }^{+}$ions listed in column 4. Similarly, the contributions of the $\mathrm{PbCH}_{2}{ }^{+}, \mathrm{PbCH}^{+}$, and $\mathrm{PbC}^{+}$ ions are obtained and listed in the columns indicated. In this case, there are four checks on the accuracy of the calculation at masses $216,217,218$, and 219 . Column 8 shows the differences between the sums of the computed contributions and the observed abundances of these four peaks. The agreement is entirely satisfactory, since errors are cumulative and some deviations are expected on the small peaks.

TABLE 3. Calculation of the $\mathrm{Pb}\left(\mathrm{CH}_{3}\right)^{+}$portion of the monoisotopic spectrum of tetramethyl lead for $\mathrm{\gamma O}^{\mathrm{v}} \mathrm{v}$ electrons

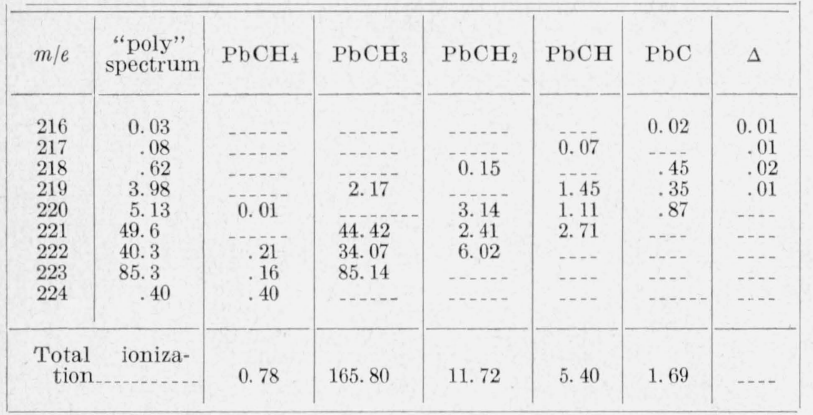

The total ionization of the isotopic ions is given at the bottom of the appropriate columns. Similar calculations were made for the $\mathrm{Pb}^{+}$, the $\mathrm{Pb}\left(\mathrm{CH}_{3}\right)_{2}{ }^{+}$ and the $\mathrm{Pb}\left(\mathrm{CH}_{3}\right)_{3}{ }^{+}$regions (the small peaks in the $\mathrm{Pb}\left(\mathrm{CH}_{3}\right)_{4}{ }^{+}$region were summed directly.) The summation values were normalized relative to the most abundant ion, $\mathrm{Pb}\left(\mathrm{CH}_{3}\right)_{3}{ }^{+}$. These values appear in columns 2 and 5 of table 4 . The abundance of the hydrocarbon ions was also normalized relative to the $\mathrm{Pb}\left(\mathrm{CH}_{3}\right)_{3}{ }^{+}$ion and is included in this table. Similar calculations on data obtained with 50-v electrons differed only in that the ratio $\mathrm{PbH}^{+} / \mathrm{Pb}^{+}=$ 0.094 was used to compute the abundance of the lead isotopes.

In tetraethyl lead, the ratio $\mathrm{PbH}^{+} / \mathrm{Pb}^{+}$was 0.88 and 0.95 , respectively, for $70-$ and $50-\mathrm{v}$ electrons as determined from the polyisotopic spectra. No dihydride was observed, and it is estimated that the ratio $\mathrm{PbH}_{2}^{+} / \mathrm{Pb}^{+}$was less than 0.002 . The mean mole percentages of the lead isotopes determined for tetraethyl lead are given in the second row of table 2 . The monoisotopic spectrum for $70-\mathrm{v}$ electrons calculated as described above appears in columns 3

:"Isotopic ions" are defined as ions identical in specie and number of constituent atoms but differing in the isotopic weight of one or more of the constituent atoms.
TABLE 4. Monoisotopic spectra of tetramethyl and tetraethyl lead for $70-v$ electrons

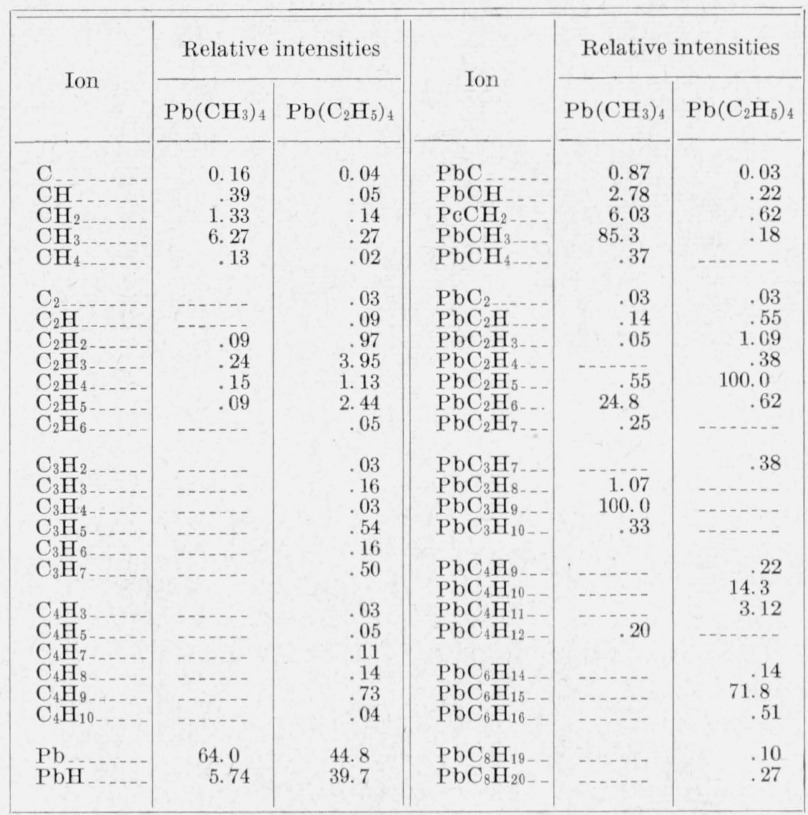

and 6 of table 4 . Twelve small peaks of doubtful origin in the mass range 69 to 101 have been omitted. These were all considerably less than 1 percent of the maximum peak.

Three peaks due to metastable transitions were also omitted. These were observed to have apparent masses of $190.4,211.2$, and 239.9 with abundances of $0.21,0.20$, and 0.25 percent, respectively, of the maximum peak. They were ascribed to the transitions $\left(295^{+}\right) \rightarrow\left(266^{+}\right)+29,\left(266^{+}\right) \rightarrow\left(237^{+}\right)+29$, and $\left(295^{+}\right) \rightarrow\left(237^{+}\right)+58$. No doubly charged ions were observed. The sensitivity of the maximum peak relative to the $n$-butane maximum peak was 1.26 at $50 \mathrm{v}$.

Monoisotopic spectra of dimethyl, diethyl, and di- $n$-butyl mercury were calculated from the polyisotopic spectra by the method used for the lead compounds. Monohydrides of the atom ions were observed in each case, and a dihydride was apparent in diethylmercury. The ratio $\mathrm{HgH}^{+} / \mathrm{Hg}^{+}$was 0.117 , 0.215 , and 0.04 , respectively, for the methyl, ethyl, and butyl compounds at $70 \mathrm{v}$. The ratio was 0.118 , 0.225 , and 0.04 for $50-\mathrm{v}$ electrons. In diethyl mercury the dihydride ratio $\mathrm{HgH}_{2}^{+} / \mathrm{Hg}^{+}$was 0.01 for both 50- and 70-v, and was estimated to be less than 0.003 for the other two compounds. Using the appropriate ratios, the mole percentages of the mercury isotopes were calculated as before. The mean values and the mean deviations for each of the compounds are given in table 5. The isotopic abundance of mercury reported by Hibbs [6] and by Nier [7] are included for comparison.

In the computation of the monoisotopic spectra of the mercury alkyls, larger errors appeared in the mercury-hydrocarbon portion of the spectrum than appeared in the case of the lead alkyls. This was 
TABLE 5. Mole percentage of mercury isotopes

\begin{tabular}{|c|c|c|c|c|c|c|c|}
\hline Mas: & 196 & 198 & 199 & 200 & 201 & 202 & 204 \\
\hline $\mathrm{Hg}\left(\mathrm{CH}_{3}\right.$ & 0.17 & 10.05 & 16.88 & 23.12 & 13.12 & 29.78 & 6.87 \\
\hline Mean deviation. & \pm .01 & \pm .02 & \pm .03 & \pm .04 & \pm .02 & \pm .06 & \pm .04 \\
\hline $\mathrm{Hg}\left(\mathrm{C}_{2} \mathrm{H}_{5}\right)_{2}$ & .18 & 10.05 & 16.86 & 23.19 & 13.05 & 29.79 & 6.91 \\
\hline Mean deviation & \pm .0 & \pm .08 & \pm .02 & \pm .09 & \pm .08 & \pm .09 & \pm .05 \\
\hline $\mathrm{Hg}\left(n-\mathrm{C}_{4} \mathrm{H}_{9}\right)$ & .16 & 10.04 & 16.86 & 23.06 & 13.15 & 29.86 & 6.89 \\
\hline Mean & \pm .04 & $\pm .0 i$ & \pm .03 & \pm .08 & \pm .07 & \pm .01 & \pm .05 \\
\hline Hibbs [6]; Hg vapor & .16 & 10.02 & 16.92 & 23.10 & 13. 22 & 29. 72 & 6.84 \\
\hline Nier [7]; Hg vapor_ & .146 & 10.02 & 16.84 & 23.13 & 13. 22 & 29.80 & 6.85 \\
\hline
\end{tabular}

undoubtedly the result of the increase in the number of isotopes, and the fact that a major portion of the total ionization in the mercury alkyls was contributed by hydrocarbon ions. For the latter reason, four additional runs were made on dimethyl mercury at pressures up to three times the normal operating pressure of the mass spectrometer. The $\mathrm{HgH}^{+} / \mathrm{Hg}^{+}$ ratio was unchanged, and no dihydride was observed. The isotopic abundance ratios were within the average deviations given for dimethyl mercury in table 5 .

The major portions of the monoisotopic spectra of the three mercury alkyls are given in table 6 for $70-\mathrm{v}$ electrons. The ion intensities are relative to the most abundant ion containing mercury since this is a more convenient basis for comparison. A number of peaks due to double ionization, metastable transition, and rearrangement are omitted from the table. Some of these are described below.

No metastable transitions were observed in dimethyl mercury. The only doubly charged ions, $\mathrm{Hg}^{++}$, were less than 0.5 percent of the $\mathrm{HgCH}_{3}^{+}$peak.

TABLE 6.-Portions of the monoisotopic spectra of dimethyl, diethyl and di-n-butylmercury for 70-v electrons

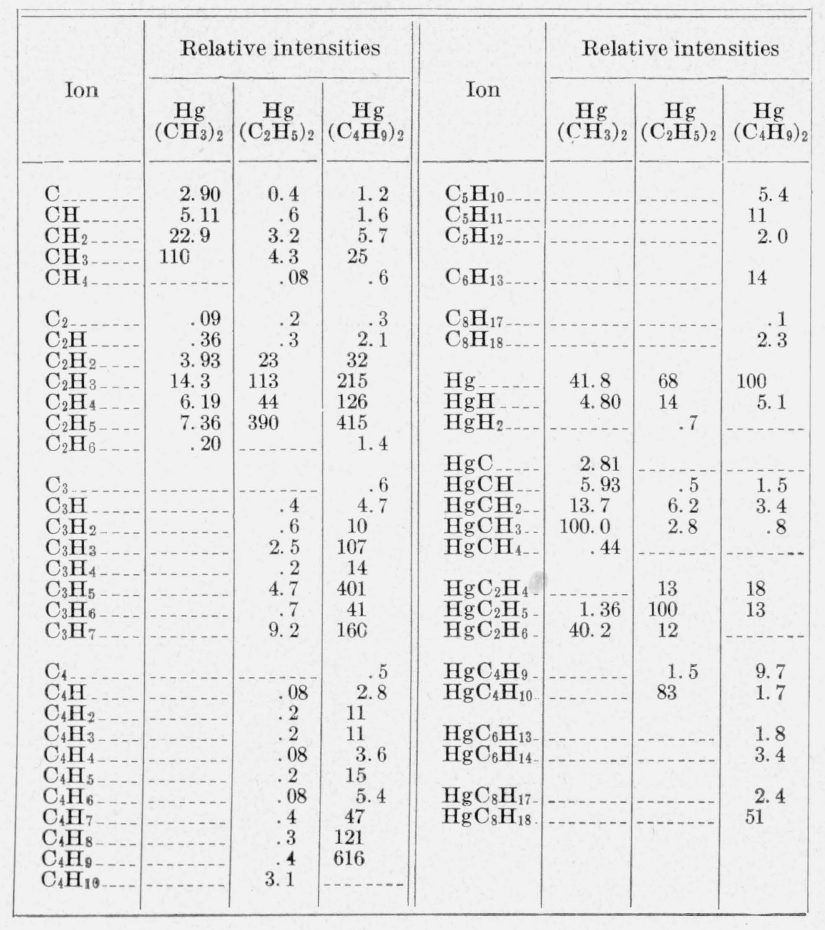

The sensitivity of the $\mathrm{CH}_{3}^{+}$ion relative to the sensitivity of the $n$-butane 43 peak was 1.1 at $70 \mathrm{v}$.

In diethyl mercury the one metastable transition resulted in an ion with an apparent mass of 25.1 and an abundance of 0.3 percent relative to the $\mathrm{HgC}_{2} \mathrm{H}_{5}^{+}$ peak. The sensitivity of the $\mathrm{C}_{2} \mathrm{H}_{5}^{+}$ion relative to the 43 peak of $n$-butane was 1.9 at $70 \mathrm{v}$.

Three metastable transition peaks were observed in dibutyl mercury with apparent masses of 37.1 , 29.5 , and 25.1 and respective percentage abundances of $0.4,1.4$, and 0.4 relative to the $\mathrm{Hg}^{+}$ion. These were attributed to the transitions: $\left(41^{+}\right) \rightarrow\left(39^{+}\right)+2$, $\left(57^{+}\right) \rightarrow\left(41^{+}\right)+16$, and $\left(29^{+}\right) \rightarrow\left(27^{+}\right)+2$. Doubly charged ions, $\mathrm{C}_{4} \mathrm{H}_{3}^{++}, \mathrm{C}_{4} \mathrm{H}_{5}^{++}$, and $\mathrm{Hg}^{++}$were observed with the respective percentage abundances $1.2,0.2$, and 2.9 relative to the $\mathrm{Hg}^{+}$ion. The sensitivity of the $\mathrm{C}_{4} \mathrm{H}_{9}^{+}$ion was of the order of 10 times the sensitivity of the 43 peak in $n$-butane.

\section{Discussion}

\subsection{Isotope Assay}

The precision of the isotope abundance measurements of lead using the tetramethyl and tetraethyl compounds is indicated by the mean deviation given in table 2. An uncertainty of 1 percent in the mole percentages of the principal isotopes and 5 percent in the rare isotope is principally the result of errors in the recording system. The small residuals in the calculations illustrated by table 3 indicate a high order of accuracy in the correction for hydrides and the computation of the isotopic abundance.

The abundances obtained using the tetraethyl lead are in good agreement with the values reported by White and Cameron for "common" lead chloride. The abundance values obtained for the tetramethyl compound differ from the values for tetraethyl lead by an amount greater than the uncertainty in the two measurements. They are more nearly in agreement with the values reported by Nier for lead obtained from galena of Joplin, Mo. Differences like this are to be expected, as commercial lead will usually be a mixture of leads from many sources but sometimes lead from one source will predominate.

Both the tetramethyl and the tetraethyl lead appear to be suitable compounds for the isotopic assay of the element. ${ }^{4}$ The higher vapor pressure of the teiramethyl lead is an advantage in the speedy removal of samples from the reservoir. Unlike the inorganic lead halides, conventional precautions for pumping out the lead alkyls are all that is required to prevent memory effects. Inlet systems without lubricated stopcocks are expected to be superior in this respect to the sample handling system used in this research.

The precision of the isotopic abundance measurements of mercury using dimethyl, diethyl, and dibutyl mercury is indicated by the mean deviations given in table 5. The estimated uncertainty in the abundance of the principal isotopes is 1 percent of the value. The uncertainty in the rare isotope is

1 After this paper was written, Collins, Freeman, and Wilson [12] reported the use of lead tetramethyl for mass spectrometric analyses in the isotopic lead use of lead tetramethyl for mass spectrometric 
about 10 percent of the value. The agreement between the values obtained by using the mercury alkyls is very good, and they agree well with values obtained by Hibbs and by Nier, using mercury vapor. The consistency of the results for three very different molecules shows that the correction for the contributions of the hydride ions can be made with precision. It also shows that in these measurements made with different instruments and techniques there is no evidence of instrumental selectivity for ions of different mass. As our measurements were made by electrostatic scanning, any selectivity would tend to make the heavier isotope peak too small. Hibbs used magnetic scanning, which is not subject to this effect, whereas Nier calibrated for discriminative effects with carefully made synthetic isotope mixtures of $\mathrm{A}^{36}$ and $\mathrm{A}^{40}$.

Schaeffer [10] has recently published data showing large discrimination effects in a $60^{\circ}$ mass spectrometer when the isotopes of krypton were measured using electrostatic scanning. The present results with mercury confirm previous evidence that in the $180^{\circ}$ Consolidated mass spectrometer this discrimination is negligible for most isotope measurements. Berry [11] has also demonstrated this to be the case over a wide range of ion-accelerating voltage when the ions are formed with little kinetic energy.

\subsection{Dissociation Probabilities}

The mass spectrum of tetramethyl lead is like that of 2,2-dimethylpropane [2] in several respects. The relative abundance of the molecule ion is small, and the most probable dissociation is the loss of one methyl group. However, the large abundance of the $\mathrm{Pb}^{+}$ion (fortunate from the standpoint of isotopic assay) illustrates a marked difference resulting from the substitution of a heavy metal atom for the central carbon atom of the hydrocarbon.

The ratio $\mathrm{Pb}\left(\mathrm{CH}_{3}\right)_{3}^{+} / \mathrm{Pb}\left(\mathrm{CH}_{3}\right)_{4}^{+}$observed in this research is about ten times that reported by Hipple and Stevenson [4]. This is to be expected, however, since the ion source used by Hipple and Stevenson operated at nearly room temperature compared to the operating temperature of $285^{\circ} \mathrm{C}$ for the source used in this study.

The relative probabilities of dissociating one and three methyl groups are nearly equal, whereas the probability of dissociating two methyl groups is much smaller. Ion peaks resulting from the dissociation of one or more hydrogen atoms from the lead-methyl groups are small, as are the hydrocarbon peaks. This results in a comparatively simple spectrum, with the $\mathrm{Pb}\left(\mathrm{CH}_{3}\right)_{3}^{+}$and the $\mathrm{Pb}\left(\mathrm{CH}_{3}\right)^{+}$ions being the principal contributors to the total ionization.

The ions $\mathrm{PbH}, \mathrm{HPbCH}_{3}, \mathrm{HPb}\left(\mathrm{CH}_{3}\right)_{2}$, and $\mathrm{HPb}$ $\left(\mathrm{CH}_{3}\right)_{3}$ involve a rearrangement of $\mathrm{H}$ atoms in the ionization process. The very small hydrocarbon peaks containing two carbon atoms also involve a rearrangement of two methyl groups in the ionization process. The relative intensities of the latter ions indicate that they are not the result of a hydrocarbon impurity.
Except for the low abundance of the molecule ion, which is almost always associated with this type of symmetry, the mass spectrum of tetraethyl lead is quite unlike that of its carbon analog, 3,3-diethylpentane [2]. It is, however, qualitatively similar to the tetramethly lead spectrum. The relative probabilities of dissociating one and three ethyl groups is reversed, the latter being the more probable in the tetraethyl compound. As in tetramethyl lead, a relatively simple spectrum is obtained with the $\mathrm{Pb}\left(\mathrm{C}_{2} \mathrm{H}_{5}\right)^{+}$and the $\mathrm{Pb}\left(\mathrm{C}_{2} \mathrm{H}_{5}\right)_{3}^{+}$ions contributing the major portion of the total ionization. The abundance of fragment ions involving dissociation of hydrogen atoms or methyl radicals is relatively small.

As in tetramethyl lead we ind fragment ions containing one additional $\mathrm{H}$ atom. These are $\mathrm{PbH}$ $\mathrm{HPbC}_{2} \mathrm{H}_{5}, \mathrm{HPb}\left(\mathrm{C}_{2} \mathrm{H}_{5}\right)_{2}$, and $\mathrm{HPb}\left(\mathrm{C}_{2} \mathrm{H}_{5}\right)_{3}$; and the first and third are much more abundant than the analogous ions in the tetramethyl spectrum. The presence of hydrocarbon ions containing three and four carbon atoms indicates a rearrangement of ethyl radicals in the ionization process analogous to the formation of $\mathrm{C}_{2}$ ions from methyl radicals in the tetramethyl spectrum. However, unidentified trace impurities may contribute to these peaks.

The mass spectra of the three mercury alkyls are significantly different from the lead alkyls in several respects. A large fraction of the total ionization in each case is contributed by the hydrocarbon ions, the maximum peaks in each case being the hydrocarbon ions, $\mathrm{CH}_{3}^{+}, \mathrm{C}_{2} \mathrm{H}_{5}^{+}$, or $\mathrm{C}_{4} \mathrm{H}_{9}^{+}$. Also, the abundance of the molecule ions is of the same order of magnitude as that of the base peak. This is a distinct difference between the mercury and the lead compounds.

As in the lead alkyls, monohydrides of the metal ions were observed. A dihydride, $\mathrm{HgH}_{2}^{+}$was observed in the case of diethylmercury only. A number of spectra of dimethyl-mercury obtained over a range of pressures up to three times the normal operating pressure of the mass spectrometer showed that the ratio $\mathrm{HgH}^{+} / \mathrm{Hg}^{+}$was constant. This is evidence that the metal hydride is formed by intramolecular rearrangement rather than by recombination. The abundance of the hydrides in the mercury-alkyl portions of the spectra was less accurately measured but also appeared to be constant over the pressure range. Identification of some of these ions is uncertain, particularly in the dibutyl mercury because of the low relative abundance of the mercury alkyl groups. The low abundance of $\mathrm{HgC}_{4} \mathrm{H}_{9}^{+}$in the dibutyl spectrum is unexpected, for in this respect it is unlike the other two compounds.

In addition to the hydrides, hydrocarbon peaks involving rearrangements are observed in all three spectra. As in the lead compounds, methyl, ethyl, and butyl radicals apparently rearrange to form $\mathrm{C}_{2}$, $\mathrm{C}_{4}$, and $\mathrm{C}_{8}$ hydrocarbons, respectively. However, identification is uncertain because of the small size of the peaks and the possible contributions of suspected hydrocarbon impurities. 


\subsection{Chemical Analysis}

The sensitivity of the most abundant ions in tetramethyl lead and tetraethyl lead is of the same order of magnitude as the sensitivity of the most abundant ion in most hydrocarbons. A commercial gasoline commonly contains a few hundredths of a mole percent of tetraethyl lead. Consequently, the peaks corresponding to the $\mathrm{PbC}_{2} \mathrm{H}_{5}^{+}$ion will be a few hundredths of a percent of the maximum hydrocarbon peak. The 21-102 Consolidated mass spectrometer has a sensitivity of about 0.01 percent of the maximum peak, and this is scarcely adequate for quantitative measurements. The new 21-103 instrument, however, has about 10 times this sensitivity. A preliminary test with a 21-103 instrument on a gasoline containing $2.5 \mathrm{ml}$ of tetraethyl lead per gallon, or about 0.045 mole percent, gave $\mathrm{PbC}_{2} \mathrm{H}_{5}^{+}$peaks that could be measured with a precision of about 2 percent. Further investigation of this point is under way in this laboratory. Instruments made by the General Electric Co. and by the Metropolitan Vickers Co. have comparable sensitivity and are probably of equal value for such analyses.

\section{References}

[1] Mass spectrometry, a review by J. A. Hipple and M. Shepherd, Anal. Chem. 21, 32 (1949).

[2] API Tables of Mass Spectral Data (Carnegie Institute of Technology, Pittsburgh, Pa., 1948).

[3] F. W. Aston, Mass spectra and isotopes, 2d ed. (Longmans, Green and Co., New York, N. Y.).

[4] J. A. Hipple and D. P. Stevenson, Phys. Rev. 63, 121 (1943).

[5] A. O. Nier, R. W. Thompson, and B. F. Murphey, Phys Rev. 60, 112 (1941).

[6] R. F. Hibbs, USAEC unclassified report Y-472.

[7] A. O. Nier, Phys. Rev. 79, 450 (1950).

[8] V. H. Dibeler and F. Cordero, J. Research NBS 46, 1 (1951) RP2167.

[9] J. R. White and A. E. Cameron, Phys. Rev. 74, 991 (1948).

[10] A. O. Schaeffer, J. Chem. Phys. 18, 1681L (1950).

111] C. E. Berry, Phys. Rev. 78, 597 (1950).

[12] C. B. Collins, J. R. Freeman, and J. T. Wilson, Phys Rev. 82, 966 (1951).

Washington, May 3, 1951 